\title{
Resistência de união à dentina de resinas compostas associadas a sistemas adesivos com e sem carga
}

\section{Bond strength of resin composites to dentin associated to filled and unfilled adhesive systems}

\author{
Jandyra A. YOUSSEF* \\ Miriam Lacalle TURBINO** \\ Michel Nicolau YOUSSEF*** \\ Edmir MATSON****
}

\begin{abstract}
YOUSSEF, J. A.; TURBINO, M. L.; YOUSSEF, M. N.; MATSON, E. Resistência de união à dentina de resinas compostas associadas a sistemas adesivos com e sem carga. Pesqui Odontol Bras, v. 15, n. 2, p. 157-160, abr./jun. 2001.

Este trabalho analisou in vitro duas marcas de adesivos de quarta geração do sistema simplificado (Optisolo - Kerr, com carga, e Single Bond - 3M, sem carga) e duas marcas de resinas compostas (Prodigy - Kerr e Z100 - 3M), com o objetivo de verificação da adesividade na dentina. Oitenta corpos-de-prova, confeccionados a partir de molares humanos extraídos, foram incluídos em resina acrílica e desgastados até exposição de dentina no sentido longitudinal, e divididos em 4 grupos. Cones de resina composta foram aderidos a estes corpos-de-prova precedidos dos sistemas adesivos, seguindo a orientação dos fabricantes. Os corpos-de-prova foram submetidos a teste de tração numa máquina de ensaios Universal Mini-Instron 4442, a uma velocidade de $0,5 \mathrm{~mm} / \mathrm{min}$. Os resultados obtidos foram transformados em MPa de acordo com a área de adesão e submetidos a análise estatística pela ANOVA. Pelos resultados obtidos, concluiu-se que houve diferença estatisticamente significante $(\mathrm{p}<0,01)$ entre os adesivos $(\mathrm{F}=7,24)$, sendo que o adesivo Optisolo $(\mathrm{m}=11,03 \pm 4,23)$ apresentou maior resistência de união que o Single Bond $(\mathrm{m}=8,37 \pm 4,54)$. Entre as duas resinas $(F=0,43)$, não foi detectada diferença estatisticamente significante $(p>0,05)$.
\end{abstract}

UNITERMOS: Adesivos dentinários; Resinas compostas.

\section{INTRODUÇÃO}

A retenção das resinas compostas ao esmalte é um procedimento eficiente e consagrado devido ao uso de condicionamento ácido e adesivos. O mesmo não acontece com a dentina, em função de suas características, que não favorecem o embricamento micromecânico como ocorre com o esmalte condicionado ${ }^{11}$.

O inter-relacionamento da dentina com a polpa ocorre através de túbulos que vão desde a polpa até o limite amelodentinário, apresentando em seu interior a presença constante do fluido tissular dentinário. Esses túbulos apresentam número e tamanho variáveis, e em conseqüência, a quantidade de dentina peri- e intertubular é diferente num mesmo dente, dependendo da região e da profundidade da dentina considerada. Essas variações da dentina interferem na permeabilidade das diversas regiões, podendo comprometer a ade- sividade $^{3,7}$. Os sistemas adesivos que apresentam ótimo comportamento no esmalte, ainda são questionados quanto à sua eficiência em dentina. Estes sistemas vêm apresentando, ao longo do tempo, várias alterações, tanto em suas composições quanto em suas apresentações. Tanto os adesivos convencionais - ácido, "primer" e adesivo em embalagens isoladas - quanto os simplificados - ácido numa embalagem e "primer" e adesivo em único frasco, visam a obtenção da camada híbrida ${ }^{4,5}$, fundamento indispensável para a retenção micromecânica.

Vários fatores podem contribuir para a eficiência ou não dos adesivos. Dentina seca ou úmi$\mathrm{da}^{15,16}$, capacidade de penetração dos monômeros utilizado ${ }^{10,20}$, contração de polimerização das resinas compostas ${ }^{8,12}$, grau de polimerização das resinas $^{4,17}$, tamanho das partículas de carga da resina $\operatorname{composta}^{17}$, microfendas formadas entre a dentina

\footnotetext{
*Pós-graduanda; **Professora Doutora; ****Professor Titular - Departamento de Dentística da Faculdade de Odontologia da USP. *** Professor Associado do Departamento de Dentística da Faculdade de Odontologia da USP e Professor Titular de Dentística da Faculdade de Odontologia da Universidade Mogi das Cruzes.
} 
YOUSSEF, J. A.; TURBINO, M. L.; YOUSSEF, M. N.; MATSON, E. Resistência de união à dentina de resinas compostas associadas a sistemas adesivos com e sem carga. Pesqui Odontol Bras, v. 15, n. 2, p. 157-160, abr./jun. 2001.

condicionada e penetrada pelo adesivo e a dentina não desmineralizada, falha essa denominada de nanoinfiltração ${ }^{9}$ e a espessura da camada híbri$\mathrm{da}^{20}$.

O objetivo desta pesquisa foi avaliar, in vitro, a efetividade de dois sistemas adesivos simplificados, um com carga (Optibond Solo - Kerr) e outro sem carga (Single Bond - 3M), sobre a dentina condicionada, utilizando duas resinas compostas híbridas, procedentes de diferentes fabricantes (Prodigy - Kerr e Z100 - 3M).

\section{MATERIAL E MÉTODO}

Foram utilizados 80 corpos-de-prova confeccionados a partir de terceiros molares humanos extraídos, livres de cárie, incluídos em resina acrílica e desgastados, em sua face vestibular, até a exposição da dentina no sentido longitudinal. Esses dentes foram divididos em 4 grupos de 20 unidades. Cones de resina composta foram aderidos à dentina desses dentes precedidos da aplicação dos sistemas adesivos, seguindo a orientação de cada fabricante. Foram combinadas duas resinas compostas (Prodigy - Kerr e Z100 - 3M) com 2 adesivos dentinários (Optibond Solo - Kerr e Single Bond $3 \mathrm{M})$.

Após o condicionamento ácido, cada corpo-deprova era fixado em um suporte metálico. Uma matriz de Teflon com formato interno cônico também foi fixada sobre cada corpo-de-prova de maneira que o adesivo e a resina pudessem ser aplicados sobre a dentina. Nos grupos 1 e 3, foi aplicado Optibond Solo, com o auxílio de uma ponta Microbrush, sendo em seguida polimerizado por 20 segundos (Ultralux - Dabi-Atlante). Nos grupos 2 e 4, foi aplicado o Single Bond, também com as pontas Microbrush, em duas camadas, com ligeira secagem por 2 segundos com jato de ar e em seguida polimerizado por 10 segundos. As resinas foram inseridas e fotopolimerizadas em 3 incrementos iguais e fotopolimerizadas por 40 segundos cada camada.

Os corpos-de-prova foram submetidos a testes de tração numa máquina de ensaios Universal Mini-Instron 4442, a uma velocidade de $0,5 \mathrm{~mm} / \mathrm{min}$.

Após a fratura, cada corpo-de-prova era analisado com auxílio de uma lupa com aumento de 7 vezes (Magnifer FD20) para avaliar o tipo de fratura. Essas fraturas eram classificadas em Adesiva (A), quando havia destacamento total do cone de resina, Coesiva $(C)$, quando havia fratura do corpo da resina que ficava aderida à dentina, e
Adesiva/Coesiva (A/C) quando parte da resina era destacada, porém permaneciam partes da resina aderida à dentina. Os dados foram tabulados e analisados estatisticamente, pelo teste de análise de variância, com nível de significância de 1\%.

\section{RESULTADOS}

Os resultados obtidos consistiram de 80 valores de resistência de união expressos em $\mathrm{MPa}$, referentes ao cruzamento de 2 sistemas adesivos, duas resinas compostas e 20 repetições. Esses valores foram analisados estatisticamente com auxílio do software GMC 7.4. Feitos os testes preliminares, verificou-se que a distribuição dos dados era normal e homogênea, então utilizou-se a análise de variância. Os resultados dessa análise mostraram haver diferença estatisticamente significante ao nivel de $1 \%$ entre os sistemas adesivos $(\mathrm{F}=7,24)$, e pela comparação entre as médias, verifica-se que o Optibond Solo $(\mathrm{m}=11,03)$ promoveu maior resistência de união à dentina que o Single Bond $(m=8,37)$. Para o fator resinas, não foi detectada diferença estatisticamente significante $(F=0,43)$ entre as duas resinas utilizadas (Prodigy $=9,38$ e $Z 100=10,03)$. Também não foi detectada significância estatística para a interação adesivo versus resina $(F=0,37)$. As médias de cada sistema adesivo em relação às duas resinas compostas estão expressas na Tabela 1 e deram origem ao Gráfico 1. Neste gráfico, pode-se perceber que os valores do Optibond Solo foram sempre maiores que os do Single Bond e, em relação às duas resinas, o comportamento de cada adesivo foi semelhante.

Os resultados obtidos com a avaliação do tipo de fratura estão expressos na Tabela 2, onde pode-se observar que a grande maioria das fraturas ocorridas foi do tipo adesiva.

\section{DISCUSSÃO}

Muitos são os autores que estudam o comportamento das restaurações com resinas compostas, sob os mais diversos motivos. Os adesivos dentinários, além de melhorarem consideravelmente a

TABELA 1 - Médias e desvios-padrão de resistência de união da interação sistema adesivo versus resina composta (MPa).

\begin{tabular}{l|c|c}
\hline \multicolumn{1}{|c|}{ Adesivos } & Optibond Solo & Single Bond \\
\hline Resinas & $11,01 \pm 4,34$ & $7,74 \pm 4,40$ \\
\hline Prodigy & $11,06 \pm 4,24$ & $8,99 \pm 4,69$ \\
\hline \hline
\end{tabular}


YOUSSEF, J. A.; TURBINO, M. L.; YOUSSEF, M. N.; MATSON, E. Resistência de união à dentina de resinas compostas associadas a sistemas adesivos com e sem carga. Pesqui Odontol Bras, v. 15, n. 2, p. 157-160, abr./jun. 2001.

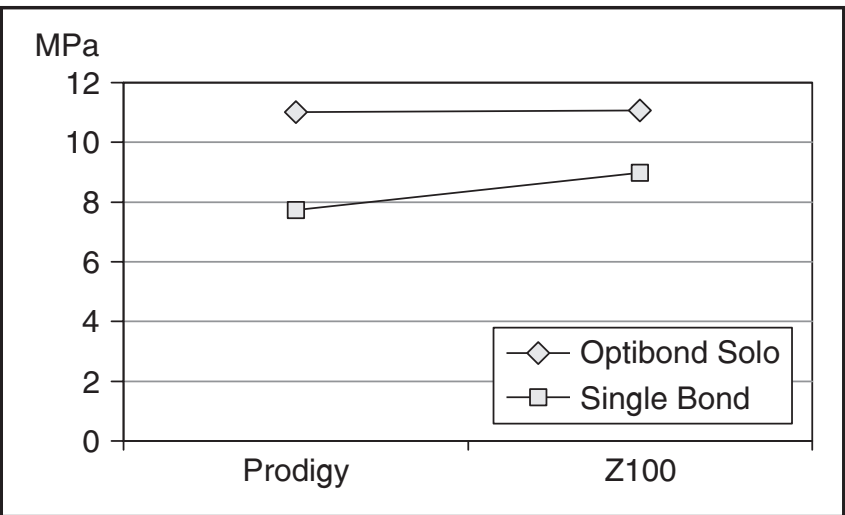

GRÁFICO 1 - Médias de resistência de união da interação sistema adesivo versus resina composta.

retenção dessas restaurações, possibilitam a conservação das estruturas dentais e promovem vedamento tanto dentinário quanto de esmalte ${ }^{1}$.

Um fator importante a ser considerado é a capacidade de penetração do adesivo em toda dentina condicionada. A dificuldade de penetração é maior em dentina seca do que em dentina úmida ${ }^{15,16}$. A dentina úmida é essencial para a efetividade dos adesivos hidrofilicos ${ }^{10,15,16}$. A dentina excessivamente úmida, por outro lado, pode provocar falhas e vazios ao longo da camada híbrida, além do incompleto vedamento dos túbulos, pois compromete a penetração da resina na dentina intratubular, pela presença de água nos túbulos dentinários e suas aberturas ${ }^{10}$. Em nosso experimento, ficou evidente a eficiência dos adesivos em meio úmido. A utilização dos dois sistemas adesivos obedece rigorosamente a especificação dos fabricantes no que diz respeito à secagem após o condicionamento ácido. Tanto no Single Bond quanto no Optibond Solo, o excesso de umidade foi removido, com um leve jato de ar. Dessa forma, não houve a possibilidade de colocação do adesivo sobre a dentina excessivamente úmida. Esta é a conduta atual unanimemente aceita ${ }^{6,7,8,9,13,15,16,19,20}$.

A adesão em dentina é muitas vezes avaliada por testes de tração e de cisalhamento, e os resultados obtidos são muito variáveis. O monômero adesivo utilizado não penetra completamente no substrato condicionado e uma zona de dentina desmineralizada e não impregnada por resina permanece entre a dentina hibridizada e a dentina inferior não alterada. A irregularidade de penetração do monômero na dentina ${ }^{20}$, associada à contração de polimerização das resinas compostas utilizadas para a restauração, pode levar a falhas que acarretam nanoinflitração ${ }^{9}$, após algum tempo, mesmo que a força de adesão inicial seja alta ${ }^{2,14}$. A contra-
TABELA 2 - Tipos de fratura observados nos corpos-de-prova após a tração.

\begin{tabular}{l|c|c|c|c|c|c}
\hline \multirow{2}{*}{ Adesivos } & \multicolumn{3}{|c|}{ Optibond Solo } & \multicolumn{4}{c}{ Single Bond } \\
\cline { 2 - 7 } Resinas & $\mathrm{A}$ & $\mathrm{C}$ & $\mathrm{A} / \mathrm{C}$ & $\mathrm{A}$ & $\mathrm{C}$ & $\mathrm{A} / \mathrm{C}$ \\
\hline Prodigy & 11 & 2 & 7 & 16 & 0 & 4 \\
\hline Z100 & 14 & 0 & 6 & 18 & 0 & 2 \\
\hline \hline
\end{tabular}

A - fratura adesiva; C - fratura coesiva; A/C - fratura adesiva/coesiva.

ção de polimerização pode ser parcialmente compensada pela técnica incremental ${ }^{6,9,12}$. Em nosso trabalho, a fotopolimerização foi realizada por esta técnica e cada camada recebeu 40 segundos de luz, com objetivo de eliminar possibilidades de polimerização insuficiente das resinas.

O estresse da contração de polimerização das resinas pode provocar alterações na camada de adesivo aplicada, dependendo da configuração cavitária, tendendo ao tracionamento do adesivo, prejudicando tanto o vedamento quanto a retenção da restauração ${ }^{18,19}$. O trabalho de PERDIGÃO et $a .^{8}$ (1996), utilizando vários sistemas adesivos, constatou através de microscopia eletrônica de varredura que nenhum deles foi capaz de prevenir completamente a formação de microfissuras na interface resina-dentina. O destacamento dos corpos-de-prova ocorria entre a zona de interdifusão e a resina fluida, em sua maioria. Em poucos casos, o destacamento foi observado na área imediatamente acima da porção superior da zona de interdifusão. Os resultados da tração em nosso experimento, conforme mostra a tabela, mostraram a força exigida para o destacamento e o tipo de fratura está de acordo com o conseguido no trabalho de PERDIGÃO et al. ${ }^{8}$ (1996), a maioria na área de interdifusão.

Uma forma de reduzir a influência da contração de polimerização sobre o adesivo aplicado é a utilização de adesivos com carga, diminuindo a formação de fendas na camada híbrida ${ }^{5}$. Como se sabe, quanto maior a quantidade de carga inorgânica, menor é a contração de polimerização ${ }^{12,13}$. Nossos resultados evidenciam que o sistema Optibond Solo, adesivo com carga, foi mais eficiente que o sistema Single Bond, sem carga, aumentando a retenção micromecânica, provavelmente em função de sua maior resistência coesiva.

\section{CONCLUSÕES}

Com base na metodologia utilizada e nos resultados obtidos, podemos concluir que: 
YOUSSEF, J. A.; TURBINO, M. L.; YOUSSEF, M. N.; MATSON, E. Resistência de união à dentina de resinas compostas associadas a sistemas adesivos com e sem carga. Pesqui Odontol Bras, v. 15, n. 2, p. 157-160, abr./jun. 2001.

1. o sistema adesivo Optibond Solo - Kerr apresentou maior resistência de união à dentina que o SingleBond - 3M;
2. não houve diferença na resistência de união à dentina entre as duas resinas utilizadas Prodigy - Kerr e Z100 - 3M.

YOUSSEF, J. A.; TURBINO, M. L.; YOUSSEF, M. N.; MATSON, E. Bond strength of resin composites to dentin associated to filled and unfilled adhesive systems. Pesqui Odontol Bras, v. 15, n. 2, p. 157-160, abr./jun. 2001.

This study analyzed in vitro two brands of one-step adhesive systems of fourth generation (Optisolo - Kerr, filled; and Single Bond - 3M, unfilled) and two composite resins (Prodigy - Kerr and Z100 - 3M), aiming at evaluating their bond strength to dentin. Eighty human extracted molars were embedded in acrylic resin and grounded until dentin was exposed in longitudinal direction. The specimens were divided in 4 groups. Composite resin cones were bonded to the specimens using the mentioned adhesive systems, following the instructions of the manufacturers. The test-specimens were submitted to tensile tests using a 4442 Universal Mini-Instron Machine with the speed of $0.5 \mathrm{~mm} / \mathrm{min}$. The results were converted into $\mathrm{MPa}$, according to the area of adhesion, and submitted to statistical analysis with ANOVA. There was significant statistical difference $(\mathrm{p}<0.01)$ between the adhesive systems $(F=7.24)$. Optisolo $(\mathrm{m}=11.03 \pm 4.23)$ showed better bond strength than Single Bond $(\mathrm{m}=8.37 \pm 4.54)$. There was no significant statistical difference $(\mathrm{p}>0.05)$ between the composites $(\mathrm{F}=0.43)$.

UNITERMS: Dentin-bonding agents; Composite resins.

\section{REFERÊNCIAS BIBLIOGRÁFICAS}

1. BRÄNNSTRÖM, M. Sensitivity of dentin. Oral Surg Oral Med Oral Pathol, v. 21, p. 517-526, 1966.

2. BURROW, M. F.; SATOH, M.; TAGAMI, J. Dentin-bonding durability after three years using dentin-bonding agents with and without priming. Dent Mater, v. 12, n. 5, p. 302-307, Sept. 1996.

3. ERICKSON, R. L. Surface interaction of dentin adhesive materials. Oper Dent, p. 81-97, 1992. [Suplemento 5]

4. FINGER, W. J.; UNO, S. Bond strength of Gluma CPS using the moist dentin-bonding technique. Am Dent J, v. 9, n. 1, p. 27-30, 1996.

5. NAKABAYASHI, N. Adhesive bonding with 4-Meta. Oper Dent, p. 125-130, 1992a. [Suplemento 5]

6. NAKABAYASHI, N. The hibrid layer: a resin-dentin composite. Proc Finn Dent Soc, v. 88, p. 321-329, 1992b. [Suplemento 1]

7. PASHLEY, D. H.; CARVALHO, R. M. Dentine permeability and dentine adhesion. J Dent, v. 25, n. 5, p. 355-372, Sept. 1997.

8. PERDIGÃO, J.; LAMBRECHTS, P.; VAN MEERBEEK, B. et al. The interaction of adhesive systems with human dentin. Am J Dent, v. 9, n. 4, p. 167-173, Aug. 1996.

9. SANO, H.; TAKATSU, T.; CIUCCHI, B. et al. Nanoleakage: leakage within the hybrid layer. Oper Dent, v. 20, n. 1, p. 18-25, Jan./Feb. 1995a.

10. SAUNDERS, W. P.; SAUNDERS, E. M. Microleakage of bonding agents with wet and dry bonding techniques. Am J Dent, v. 9, n. 1, p. 34-36, Feb. 1996.

11. SWIFT JUNIOR, E. J.; PERDIGÃO, J.; HEYMANN, H. O. Bonding to enamel and dentin: a brief history and state of the art, 1995. Quintessence Int, v. 26, n. 2, p. 95-110, Feb. 1995.

12. SWIFT JUNIOR, E. J.; TRIOLO JUNIOR, P. T.; BARKMEIER, W. W. et al. Effect of low-viscosity resins on the performance of dental adhesives. Am J Dent, v. 9, n. 3, p. 100-104, June 1996.

13. SWIFT JUNIOR, E. J.; WILDER JUNIOR, A. D.; MAY, K. M. et al. Shear bond strengths of one-bottle dentin adhesives using multiple applications. Oper Dent, v. 22, n. 5, p. 194-199, Sept./Oct. 1997.

14. TAM, L. E.; PILLIAR, M. Fracture surface characterization of dentin-bonded interfacial fracture toughness specimens. J Dent Res, v. 73, n. 3, p. 607-619, Mar. 1994.

15. TAY, F. R.; GWINNETT, A. J.; PANG, K. M. et al. Micromorphological sprectrum from overdrying to overwetting acid-conditioned dentin in water-free, acetone-based, single-bottle primer/adhesives. Dent Mater, v. 12, n. 4, p. 236-244, July 1996a.

16. TAY, F. R.; GWINNETT, A. J.; PANG, K. M.; WEI, S. H. Y. Resin permeation into acid-conditioned moist, and dry dentin: a paradigm using water-free adhesive primers. J Dent Res, v. 75, n. 4, p. 1034-1044, Apr. 1996b.

17. TURBINO, M. L. Contribuição ao estudo da microdureza Knoop de resinas compostas na região próxima à área de união à dentina. São Paulo, 1997. Tese (Doutorado) - Faculdade de Odontologia, Universidade de São Paulo.

18. VAN MEERBEECK, B.; INOKOSHI, S.; BRAEM, M. et al. Morphological aspects of the resin interdiffusion zone with different dentin adhesion systems. J Dent Res, v. 71 , n. 8, p. 1530-1540, Aug. 1992a.

19. VAN MEERBEECK, B.; YOSHIDA, Y.; VANHERLE, G. et al. A TEM study of two water-based adhesive systems bonded to dry and wet dentine. J Dent Res, v. 77, n. 1, p. 50-59, Jan. 1998.

20. YOUSSEF, M. N.; GUARALDI, E.; SATO, T. C. et al. Estudo comparativo de quatro filosofias adesivas quanto à penetração na dentina. Rev Assoc Paul Cir Dent, v. 52, n. 3, p. 236-239, maio/jun. 1998.

Recebido para publicação em 10/02/00

Enviado para reformulação em 22/12/00

Aceito para publicação em 01/03/01 